\title{
FEL-OSCILLATOR FOR FEEDING OF HIGH-GRADIENT ACCELERATING STRUCTURE ${ }^{*}$
}

\author{
C.A.Goldenberg, A.A.Kaminsky, A.K.Kaminsky", S.N.Sedykh, A.P.Sergeev, JINR, Dubna, Russia \\ N.S.Ginzburg, N.Yu.Peskov, IAP, Nizhny Novgorod, Russia
}

\begin{abstract}
Millimeter-wave FEL-oscillator is one of a possible source of RF power for accelerating structures of future linear colliders with high energy gain. JINR-IAP collaboration develops a high-efficiency single-mode FEL-oscillator with a Bragg resonator and reversed guide field. Output power of $48 \mathrm{MW}$ at the frequency of $30.6 \mathrm{GHz}$ with spectrum width $0.25 \%$ was registered in recent experiments using induction linac LIU-3000 (0.8 MeV, $200 \mathrm{~A}, 200 \mathrm{~ns})$.

In this paper we discuss technical solutions for providing of:

- frequency stability with accuracy better than $0.3 \%$;

- precise frequency coincidence of the oscillator and the accelerating structure;

- RF power transportation from the oscillator to the structure.
\end{abstract}

\section{INTRODUCTION}

Our group studies possible applications of millimeterwave free-electron laser (FEL) in oscillator configuration as a source of RF-power for high-gradient accelerating structures of two-beam accelerators [1]. The main features of developed oscillator are Bragg resonator and regime with reversed guide field [2], when cyclotron rotation of the beam electrons and their rotation imposed by helical wiggler have opposite directions. Using the electron beam of an induction linac LIU-3000 (0.8 MeV, $200 \mathrm{~A}, 200 \mathrm{~ns}$ ) the highest FEL-oscillator's efficiency of $26 \%$ was achieved at a frequency of $31 \mathrm{GHz}$, while the output power was about $35 \mathrm{MW}[2,3]$. The radiation frequency and spectrum width in a single-mode regime $(\Delta \lambda / \lambda \leq 0.25 \%)$ are very close to the values required in CLIC project [4]. But some important problems must be solved to create FEL-oscillator usable for such application.

Traditional Bragg resonator [5] consists of two mirrors separated by a smooth waveguide. You can easily have the interaction length large enough for operating of the oscillator. The quality factor of the resonator can be varied both by mirror lengths and by the distance between them.

"Work is supported by grants 97-02-16643 and 97-02-17379 of Russian Foundation for Basic Research.

"Email: alikk@ sunse.jinr.ru
The resonator has a set of longitudinal eigenmodes with integer number of half-waves between the mirrors and frequencies inside the reflectivity band of the mirrors. The frequency difference between the modes is about $0.5 \%$, so fixing one of them by proper choice of the operating regime is rather difficult taking into account inevitable jitter of beam energy and pulsed wiggler field.

Another problem in experimental realization of accelerating structure feeding is the precise frequency matching between the FEL-oscillator and the structure. Typical Q-factor of the structure is 300, so the value of $0.3 \%$ is an upper limit for admissible frequency mismatch. Central frequency of Bragg resonator is determined by the mirror corrugation period (typically one-half of wavelength), hence required precision of the mirror period can be estimated as $0.1 \%$ of $5 \mathrm{~mm}$, i.e. $5 \mathrm{mkm}$. This size tolerance is rather hard to realize. Besides the frequency variation due to the beam loading of the waveguide can break the matching. We think that the best solution of the problem is to provide a possibility of the precise tuning of the FEL-oscillator's radiation frequency.

Residual pressure in the accelerating section must be much lower than in typical induction linac. It forced us to use output windows, differential vacuum systems etc.

Technical solutions of mentioned problems are discussed in this paper.

\section{RADIATION FREQUENCY STABILIZATION}

There exists another type of Bragg resonator also proposed in [5]. It consists of two mirrors of lengths $L_{1}$ and $L_{2}$ with corrugation phase shift between them. Such resonator has only one mode inside the reflection band of the mirrors (central) and two symmetrical modes just outside the reflection band (side modes). Traditionally this type of the resonator seemed to have insufficient quality factor due to the small volume occupied by the RF field. But the proper choice of the mirrors coupling coefficient allowed us to design a resonator with the optimal Q-factor.

Dynamics of the oscillation build-up in the FEL with a two-mirror Bragg resonator with the corrugation phase shift was studied numerically. Time domain analysis taking into consideration the dispersion properties of the Bragg reflectors was used [6]. It allows us to investigate both the transient process and the stationary regime. 

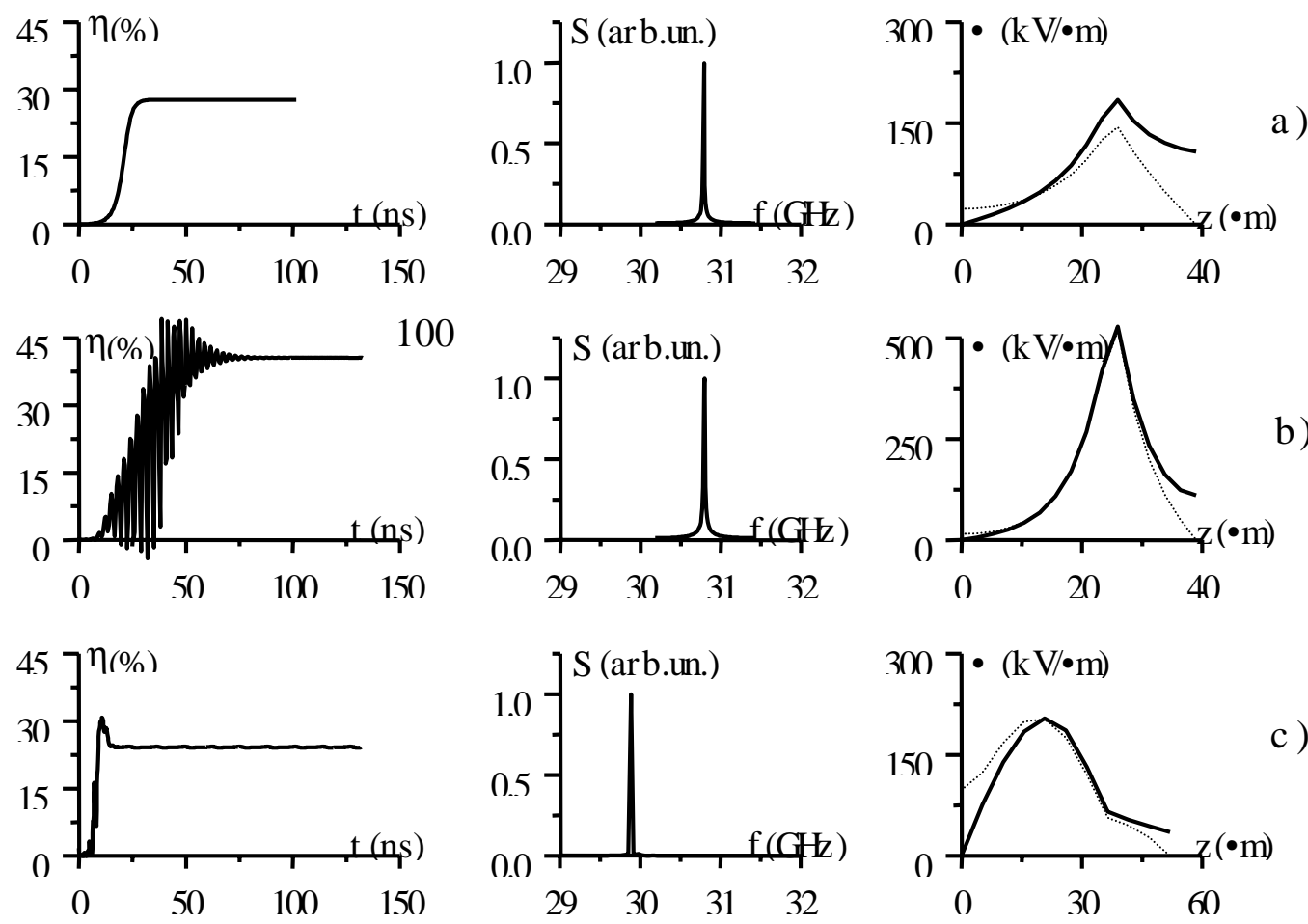

Figure 1. Numerical simulation of the oscillators with the two-mirror Bragg resonator:

Efficiency $\eta$ versus time, radiation spectrum $\mathbf{S}$ and electric field $\mathbf{E}$ distribution versus length (forward wave - solid line, backward wave - dashed line). Resonator's quality factor grows from a) to c). Fig. c) represents one of the possible operating modes.

Figure 1 presents some results of the simulation. When the Q-factor of the resonator is high enough for central mode but insufficient for side modes, the singlemode oscillation exists both in the transient regime and in the stationary regime at all the possible values of the synchronism detuning (a). If the Q-factor is large enough for the central mode and side modes too, the beating of several modes occurs in transient stage. In stationary regime the nonlinear mode competition results in the single-mode operation. The short effective interaction length (the RF field is high only near the mirrors junction) results in low sensitivity of the FEL-oscillator to the initial electron energy spread, so the oscillator efficiency can be very high (b). For too big Q-factor it is possible to operate at the central mode as well as at one of the side modes depending on the synchronism condition, but the oscillator efficiency decreases (c).

Experimental investigation of the two-mirror resonator in the optimal configuration allowed us to increase the FEL efficiency up to $35 \%$ in the single-mode regime [7].

\section{PRECISE RADIATION FREQUENCY TUNING}

Precise frequency tuning of a FEL with the two-mirror Bragg resonator may be based on the fact that the Bragg resonator eigen-frequency changes as we change the distance between the mirrors. Indeed, resonance takes

place when the phase gain of a wave travelling from one mirror to another and back is equal $2 \pi n$. But unlike "usual" mirrors the phase of a wave reflected from a Bragg mirror also depends on frequency. Let us take a Bragg resonator with the length of a smooth waveguide between the mirrors varying from zero to the corrugation period. Obviously, because each of the mirrors has many periods of corrugation, we can neglect the short section of the smooth waveguide, so changing of the length of the smooth waveguide is equivalent to the changing of corrugation phase of one mirror. At phase shift equal to zero and 2 - the resonator spectrum must be the same and coincide with the spectrum of a regular Bragg waveguide [5] (fig. 2a).

There are two resonance frequencies outside the reflection band symmetrical to the exact Bragg frequency (29.9 GHz in this figure). Distance between the two "side" frequencies is close to the reflection bandwidth and depends on the depth of the mirror corrugation. When the phase shift increases, the spectrum becomes asymmetric, and the upper "side" eigen-frequency moves inside the reflection band (fig. 2b). At the $\pi$ shift of phase we have again a well-known type of resonator [5]: the eigenfrequency coincides with the exact Bragg frequency (fig. $2 c)$. Then the eigen-frequency moves to the lower border of the reflection band (fig. $2 \mathrm{~d}$ ). Thus the tuning range is restricted by the reflection bandwidth, for the typical 


\section{Spectra for some phase shifts}

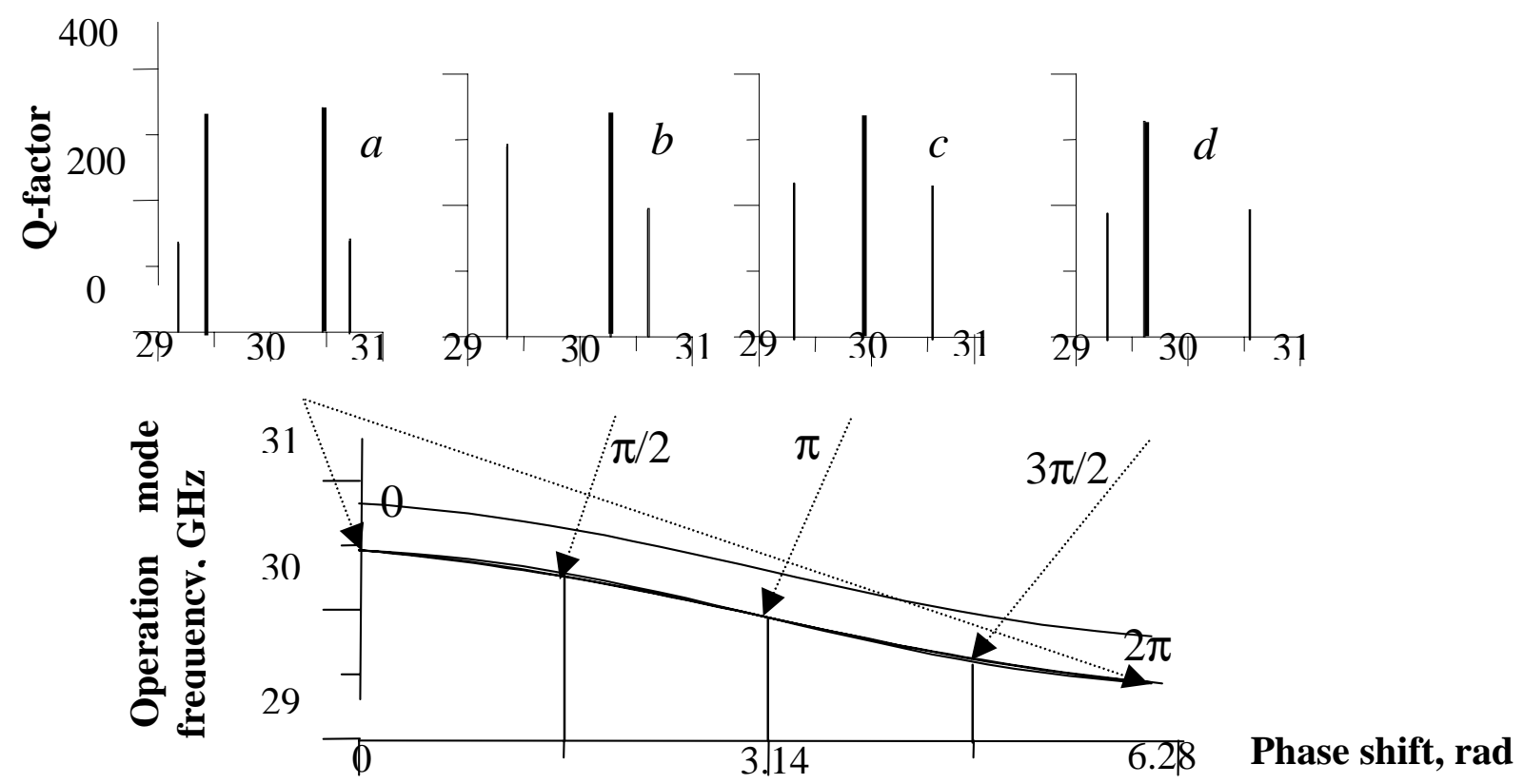

Figure 2. Spectra for some phase shifts for case $3\left(\mathrm{~L}_{1}=28, \mathrm{~L}_{2}=12 \mathrm{~cm}\right)$ and operation frequency of tunable Bragg resonators versus shift of corrugation phase for some lengths of mirrors (cm):1) $\left.\left.\left.\mathrm{L}_{1}=20, \mathrm{~L}_{2}=20,2\right) \mathrm{L}_{1}=25, \mathrm{~L}_{2}=15,3\right) \mathrm{L}_{1}=28, \mathrm{~L}_{2}=12,4\right) \mathrm{L}_{1}=30, \mathrm{~L}_{2}=10$. Period of corrugation = $0.565 \mathrm{~cm}$. waveguide radius $=1.05 \mathrm{~cm}$. counling $=0.11 / \mathrm{cm}$. modes $\mathrm{H}-\mathrm{F}$.

mirrors used in our experiments it is about $0.5-1 \mathrm{GHz}$ which equals to $(2-3) \%$ of the operation frequency $(30 \mathrm{GHz})$.

\section{EXPERIMENTAL SETUP}

Principal advantage of the FEL is the oversized waveguide in the interaction region. It allows us to use output horn and output window as large as needed for the prevention of the window breakdown. The installation includes also the mode converter of the output radiation and separated vacuum systems for induction linac and high-gradient accelerating structure. This experiment is planned to be performed at the LIU-3000. In recent experiments on the FEL-oscillator the output power of 48 MW at the frequency of $31 \mathrm{GHz}$ with spectrum width $0.25 \%$ was registered [7].

\section{CONCLUSION}

Using the two-mirror Bragg resonator with tunable phase shift between mirrors makes it possible to tune output radiation frequency of the FEL-oscillator with accuracy better than $0.3 \%$ while the radiation spectrum width do not exceeds $0.3 \%$. The radiation spectrum can be controlled by tunable narrow-band frequency filters using similar resonator with the Q-factor more than 2000. Due to the oversized waveguide the output windows can be as large as needed for preventing the RF-breakdown. So modified FEL-oscillator can be the suitable RF-source for testing the high-gradient accelerating structure at the frequency about $30 \mathrm{GHz}$ and the output power up to $50 \mathrm{MW}$.

\section{REFERENCES}

[1]. G.G. Denisov, V.L. Bratman, A.K. Krasnykh et al., "Problems of autobunching and phase stability for the TBA-driver: calculations and design for a modeling experiment" -Nuclear Instrum. and Meth., A358 (1995) 528-531.

[2]. A.K. Kaminsky, A.A. Kaminsky, V.P. Sarantsev et al., "High efficiency FEL-oscillator with Bragg Resonator operated in the reversed guide field regime." - Nuclear Instrum. and Meth., A375 (1996) 215-218.

[3]. N.S.Ginzburg, A.K.Kaminsky, A.A.Kaminsky et al., "Experimental observation of mode competition and single-mode operation in JINR-IAP millimeter-wave FEM oscillator". - Nuclear Instrum. and Meth., A407 (1998) 167-171.

[4]. CLIC, a 0.5 to $5 \mathrm{TeV}$ e+/- Compact Linear Collider CERN/PS/98009 (LP), Jean-Pierre Delahaye for the CLIC Study Team: 6th European Particle Accelerator Conference(EPAC 98), 2226.06.98, Stockholm, Sweden| and XVII International Conference on High Energy Accelerators, 7-12.09.98, Dubna, Russia

[5]. V.L.Bratman, G.G.Denisov, N.S.Ginzburg and M.I.Petelin, "FEL's with Bragg reflection resonators: autoresonance masers versus ubitrons". - IEEE J. of Quant. Electr., 1993, vol.QE-19 282-295.

[6] N.S.Ginzburg, A.S.Sergeev, N.Yu.Peskov et al.. "Mode competition and control in free-electron lasers with one and two dimentional Bragg resonators." - -Nuclear Instrum. and Meth., A375 (1996) 202-206.

[7] A.V.Elzhov, N.S.Ginzburg*, I.N.Ivanov et al. "Recent experiments on free-electron maser for two-beam accelerators" - Presented at XVII International Conference on High Energy Accelerators, Dubna, September 7-12, 1998, report c20p. 